\title{
Real-time, high-resolution ultrasonography of the vocal folds - a prospective pilot study in patients before and after thyroidectomy
}

\author{
Marek Dedecjus • Zbigniew Adamczewski • \\ Jan Brzeziński • Andrzej Lewiński
}

Received: 21 June 2010 / Accepted: 5 July 2010 /Published online: 20 July 2010

(C) The Author(s) 2010. This article is published with open access at Springerlink.com

\begin{abstract}
Purpose The aim of the study was to evaluate the functionality of vocal folds (VF) by real-time, highresolution ultrasonography (US) and to correlate the imaged features to results of laryngological examination (LE).

Methods The study group comprised 50 patients (41 females and nine males), qualified to thyroidectomy. All the patients had LE and US examination before and 2 days, 2 months, and 3 months after the surgery. We used high-resolution US imaging to identify VFs and, subsequently, a pulsed Doppler and Doppler gate to quantify the tissue displacement velocity in the vibrating VF section.

Results LE revealed unilateral VF paralysis in two patients. VF dysfunction was diagnosed in other four subjects. In simultaneously performed US examination, changes in VF displacement velocity (VFDV) were observed in ten patients. In two subjects, VFDV was below $30 \mathrm{~cm} / \mathrm{s}-$ patients with VF paralysis, diagnosed in LE. In a further eight cases, we observed VFDV decrease by $50 \%$, comparing to preoperative values. Both US-imaging and
\end{abstract}

This work was presented on the fourth Biennial ESES Congress May $13-15,2010$.

M. Dedecjus $(\bowtie) \cdot J$. Brzeziński

Department of General, Oncological and Endocrine Surgery,

Medical University of Lodz,

Polish Mother's Memorial Hospital—Research Institute,

No 281/289, The Rzgowska St,

93-338 Lodz, Poland

e-mail: mdedecjus@wp.pl

Z. Adamczewski · A. Lewiński

Department of Endocrinology and Metabolic Diseases,

Medical University of Lodz,

Polish Mother's Memorial Hospital-Research Institute,

Lodz, Poland
LE, performed after the 3-month follow-up, confirmed the transitional character of the above-mentioned pathologies. Conclusions US imaging of the VFs correlated with LE results, while being a minimally invasive, easily reproducible, and inexpensive method of examining VF functionality. Thanks to many recording options, it may soon become a perfect tool for an early identification of postoperative VF dysfunction with its later monitoring. To our knowledge, it is the first application of US and Doppler gate modes for VFDF quantification; however, an analysis on a larger group of patients is necessary to standardize the technique.

Keywords Thyroid surgery - Vocal folds .

Ultrasonography $\cdot$ Pulsed doppler $\cdot$ Doppler gate

\section{Introduction}

Vocal fold (VF) vibrations periodically interrupt the glottal airflow, generating acoustic signals, perceived as voice sounds [1]. The VF can be divided into the "cover" and the "body" [2]. The cover includes mucosa and the superficial layer of lamina propria, called the Reinke's space. The body consists of vocal ligament and vocal muscle. Functionally, the vibration of VF confined mainly to the cover and the mucosal wave propagated vertically from the lower to the upper margin of the VF [3, 4]. Changes in stiffness or tension in the VF alters the mucosal wave and laryngeal muscles play a key role in its regulation. There are three groups of intrinsic laryngeal musculature - the abductors, adductors, and tensors. The only abductor of the larynx is the posterior cricoarytenoid muscle, and it is innervated by the recurrent laryngeal nerve (RLN). The adductors are composed of the lateral cricoarytenoid muscle, interarytenoid muscle, oblique arytenoid muscles, and thyroarytenoid muscles. 
Innervation of the adductors is again supplied by the RLN. The tensors are composed of mainly the cricothyroid muscle, which is innervated by the external branch of the superior laryngeal nerve (EBSLN), and to a lesser extent by the thyroarytenoid muscles. The cover is not directly innervated and the mucosal wave is an effect of passive movements of the true VF. However, phonation is regulated by RLN and EBSLN and, of course, by the level of the airflow and of the subglottal pressure.

Vocal disability has always been considered an uncomfortable complication of thyroidectomy. Hoarseness, vocal fatigue, and low pitch are relatively frequent voice-related patient complaints after thyroid surgery. The presence of these voice disturbances has led some endocrine surgeons to advocate a laryngoscopic examination before and after thyroid operations in all the operated patients.

Laryngoscopy was postulated as the golden standard test of vocal cord mobility in thyroidectomy undergoing patients. This examination can demonstrate a paralysis of the inferior laryngeal nerve, as well as possible anatomic or functional variations after previous cervical or thoracic surgery.

However, laryngoscopy is not a common practice for many endocrine surgery teams because it is time-consuming, imposes extra costs, and is, in general, an unpleasant experience for patients. Therefore, there is a clinical need to develop a simple, inexpensive, and non-invasive technique of VF function analysis.

The aim of this study was to find an in vivo, non-invasive method to quantify the VF functionality with minimal unpleasant effects for examined patients. Considering the increasing precision and accessibility of US equipment, we decided to apply a commercially available US system for VF functionality evaluation.

\section{Patients and methods}

The study group comprised 50 patients (41 females and nine males), qualified to thyroidectomy for multinodular goiter (20 patients), toxic goiter (12 patients), thyroid cancer (10 patients), and Graves' disease (eight patients). All the patients had US examination, performed before and 2 days after thyroidectomy. Simultaneously, laryngological examination (LE) was applied. When any VF disorder was revealed, additional examinations were done 2 and 3 months after the thyroidectomy.

US imaging was used to identify the morphology of VF and pulsed Doppler and Doppler gate to quantify tissue displacement velocity in the vibrating section of VF. The study was performed with a commercially available, highresolution US scanner (Toshiba Aplio XG) with a 7.2-14-MHz linear array transducer (PLT-1204AX). The patients were recruited from the Department of General, Oncological, and
Endocrine Surgery. All of them signed their informed consent, and the Ethics Committee of the Polish Mother's Memorial Hospital, Research Institute, in Lodz approved the study protocol.

During US scanning, the patients were in recumbent position and asked to utter a sustained vowel "aa" that corresponded to the individual's most comfortable pitch. The viewing window was high enough to detect the vibratory VF part in all of the patients. VF vibration was estimated in the horizontal view. US probe was placed horizontally in the middle of the thyroid lamina on one side because the true VF is localized on this level plane. US B scan was carried out first to localize vibrating VF (Fig. 1), and then, the system was switched to pulsed Doppler and the Doppler gate was set on the VF vibrating part (Fig. 2). Then, the range of tissue motion velocity was recorded and analyzed (Fig. 3).

\section{Results}

LE, performed in the investigated group, revealed unilateral VF paralysis in two patients, while some disturbances of VF functionality were reported in four other patients. LE performed 3 months later confirmed the transitional character of the dysfunctions.

In the real-time $\mathrm{B}$ scan of $\mathrm{VF}$, the adduction and abduction motion of $\mathrm{VF}$ reflected on the movement of arytenoid cartilages and vocal ligaments. VF cover was not clearly visualized in the US images. By applying Doppler gate to the motion in that area, very strong

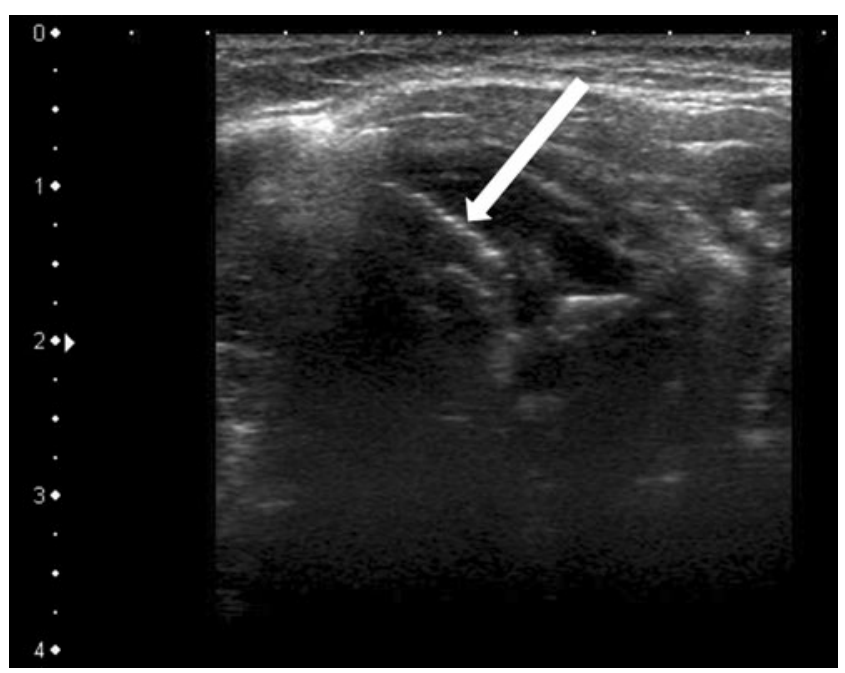

Fig. 1 The first step of the technique is to localize VF by use of Bmode US. US probe is placed horizontally in the middle of the thyroid lamina on one side. During phonation, the VF is vibrating, so it is relatively easy to localize it. The white arrow indicates VF. As a matter of fact, the arrow indicates false VF (the body) because the true $\mathrm{VF}$ (the cover) is not well visible in US 


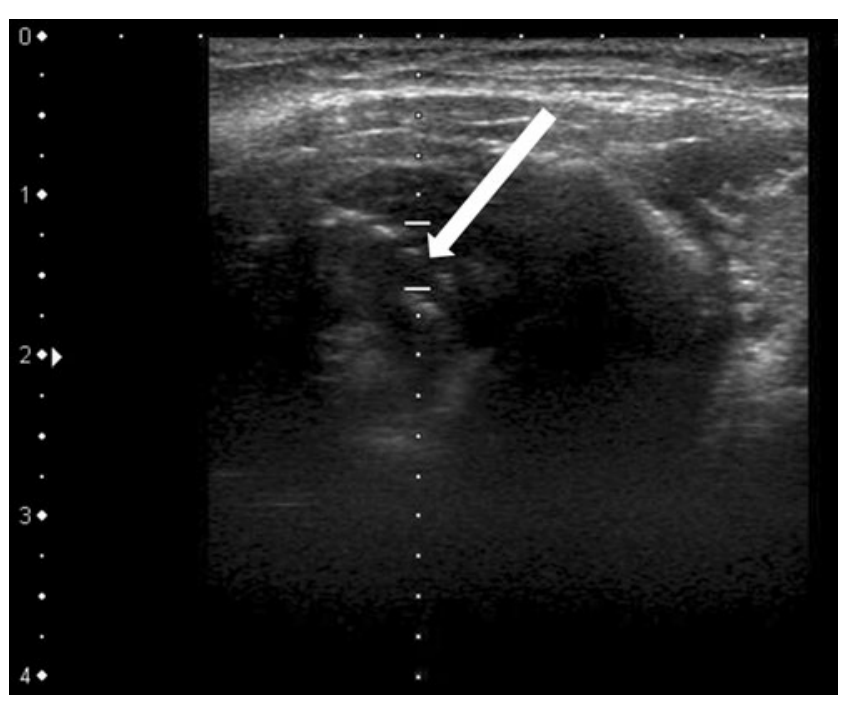

Fig. 2 The second step of the technique is to switch the system to pulsed Doppler mode and to set the Doppler gate on the vibrating section of VF. The white arrow indicates Doppler gate set on vibrating section of $\mathrm{VF}$

signals were observed, corresponding to tissue motion in the vibratory area of VF during phonation (Fig. 3). When the voice ceased, in quiet respiration position, the signals immediately disappeared.

The signals apparently came from VF cover fluctuations toward or away from the US scan head. The recorded VF displacement velocity (VFDV) ranged from 65 to $140 \mathrm{~cm} / \mathrm{s}$ for patients before operation, phonating at their most comfortable pitch and intensity. In two patients after thyroidectomy, VFDV, measured by Doppler gate on one
VF was below $30 \mathrm{~cm} / \mathrm{s}$ (Fig. 4). Those were the patients with unilateral VF paralysis, diagnosed in LE. In eight other patients, VFDV was below $50 \%$ of the level recorded before operation. That group included all four patients with VF disturbances, diagnosed in LE. The proposed criterion $(<50 \%$ decrease of VFDF) is not definitive, although it has good sensitivity, specificity (100\% and $95.7 \%$, respectively), and positive and negative predictive values $(100 \%$ and $60 \%$, respectively). Three months after thyroidectomy, VFDV returned, in all the cases, to preoperative values (Fig. 5).

\section{Discussion}

Recently, US imaging has become a very powerful diagnostic tool, especially in scanning head and neck regions [4]. Taking into consideration its non-invasive nature and minimal disturbance to normal voice production, US imaging should be an ideal tool to study VF vibration.

However, only a few studies have employed US imaging to investigate vibrating VFs [5, 6]. Although several reports have demonstrated US B-mode imaging of the larynx, including VF movements [4, 7-9], there have been very few reports describing the use of US imaging to study VF vibrations [5, 6]. It results from the lack of US imaging data on the multilayer structures of the VF and from the fact that the frame rate of US imaging is far below the vibration frequency of the VFs. In the present study, we applied a pulsed Doppler and a Doppler gate to analyze VFDV. Ultrasound imaging with Doppler gate has been used extensively as a non-invasive tool for blood flow quantification; however, to
Fig. 3 The third step of the technique is to record and analyze registered Doppler spectrum and measure VFDV. The white arrow indicates Doppler gate set on vibrating section of $\mathrm{VF}$

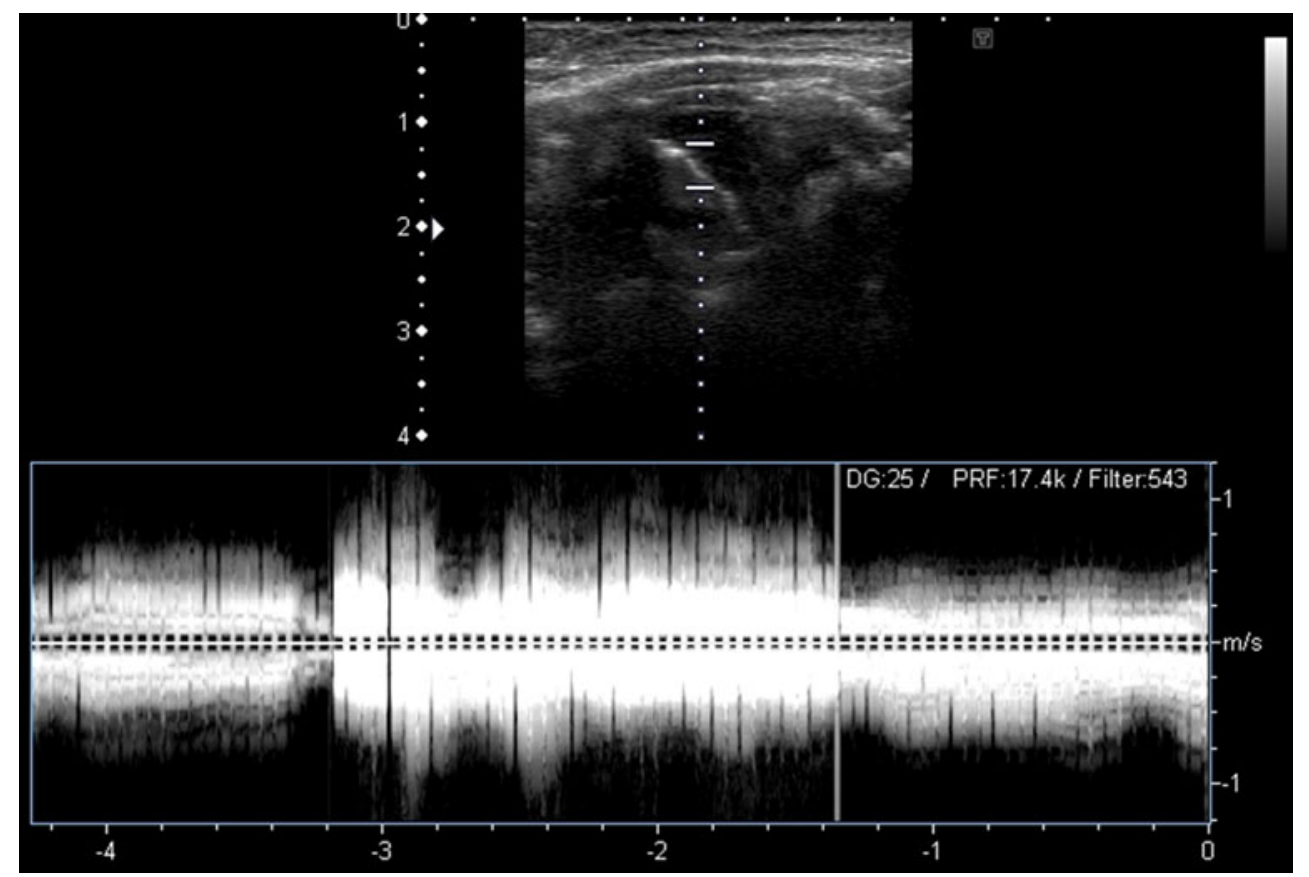


Fig. 4 Doppler spectrum of the paralyzed VF (confirmed in LE) in patient 2 days after thyroidectomy

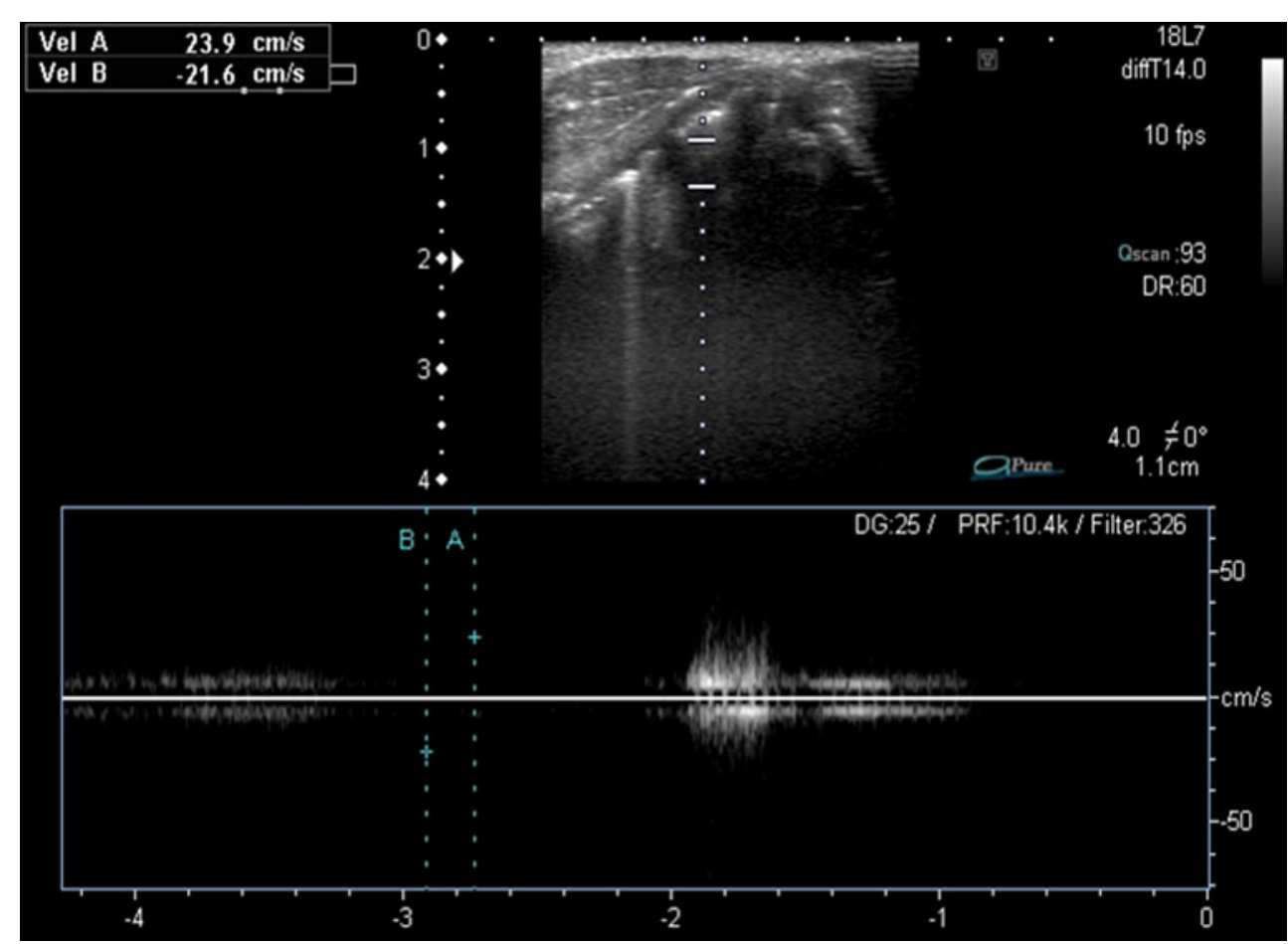

our knowledge, this was the first attempt to apply it for detection of high-frequency vibratory movements of the VFs.

Ooi et al. reported the application of CDI to identify VF palsy, based on image pattern asymmetry [10]. In a very interesting study, Shau et al. used CDI to estimate mucosal wave velocity; however, his technique was rather sophisticated [6]. In a recently published study, VF vibration physiology was studied using B-mode imaging and Echo-particle image velocimetry [11]. The authors proved that, thanks to their technique, the vibratory pattern of the VF body could be observed in human subjects under normal phonation [11].
Fig. 5 Doppler spectrum in the same patients after 3-month follow-up

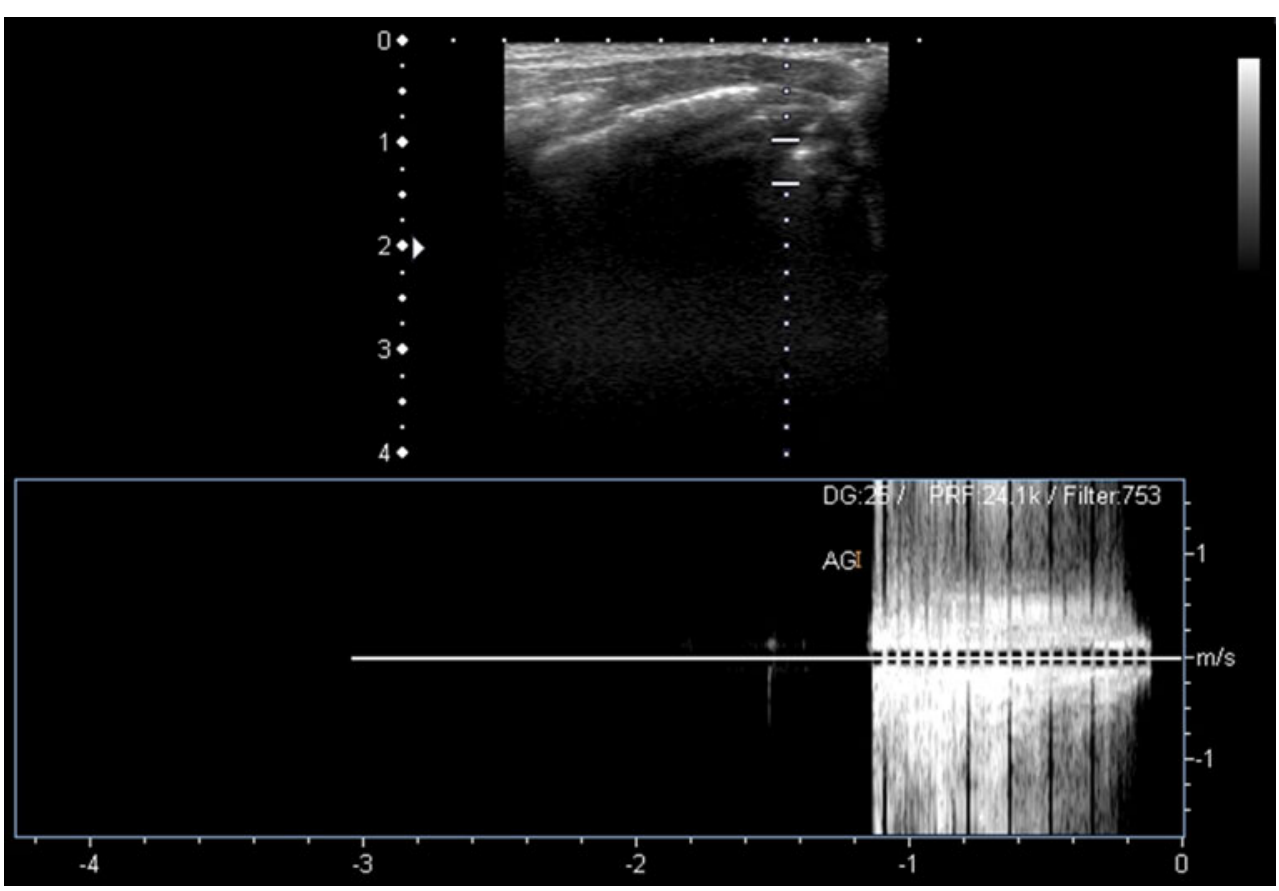


The first step of our technique was to localize VF in US B mode (Fig. 1). Calcifications of the trachea did not substantially hinder US energy transmission, and vibrating VF localization was possible even in two patients with large volume goiters $(>100 \mathrm{ml})$. During scanning, the US probe was placed laterally and horizontally. Vibrating VF was localized and the system was switched to pulsed Doppler mode (Fig. 2). Then, the Doppler gate was set on the vibrating VF. During sustained phonation, mucosa vibrations generated Doppler spectrum that could be subsequently registered and quantitatively assessed (Fig. 3). Then, the procedure was repeated on contralateral VF. Considering the position of the US probe and Doppler gate localization on the vibrating VF, the measured velocity corresponds, in our opinion, to mucosal wave velocity (MWV).

The values of MWV, measured by our technique, were in concordance with data from literature. However, further studies should be conducted to verify the conformity between MWV, measured by our technique and assessed by other, generally accepted methods. MWV correlates with the elastic properties of the laryngeal mucosa and can often differentiate normal phonation from pathology. Although it is well-known that VF stiffness greatly affects its vibratory movement in phonation, in vivo studies the mechanical properties of human VFs are rather limited $[12,13]$. The measurements were invasive and made during laryngeal surgery. Noninvasive measurements of human VF stiffness were focused on the propagation of mucosal wave, observed from the oral side $[14,15]$.

In our study, an important VFDV decrease was noted in 10 subjects on the second day after thyroidectomy. In patients with unilateral VF paralysis, diagnosed in LE, the VFDV was below $30 \mathrm{~cm} / \mathrm{s}$. Further examinations, performed 2 and 3 months after the operation, confirmed the transitional character of the disturbances and VFDV returned to its preoperative values.

Ooi et al. used for the first time the pulsed Doppler (CDI) to identify VF palsy, based on the asymmetry of color flow patterns [10]. Moreover, in echoglottography, VF paralysis can be identified, which alters the symmetry of VF vibrations $[10,16,17]$.

We have been trying to use the CDI to investigate VF functionality, but it was, sometimes, rather difficult to simultaneously visualize VF and analyze its symmetry, particularly in large goiters. Therefore, in our technique, we analyzed each VF separately. Moreover, it is easy to quantify VFDV since motion velocity assessment is a standard Doppler gate option.

VFDV quantification allowed us to reveal an important decrease of MWV after thyroid operation in eight patients. Although we were not able to define the nature of the observed disturbances, the US-investigated group comprised all the patients with VF disturbances, diagnosed in LE.
Paralysis of the EBSLN might have been, at least in part, responsible for the above-mentioned dysfunctions. Postintubation VF's dysfunction could be another possible explanation. Therefore, investigation of a group of patients before and after general anesthesia with endotracheal intubation for other operation than thyroidectomy should be performed, to rule out effects of endotracheal tube and general anesthesia.

VFDV quantification is very important, not only for the diagnosis of VF dysfunction but also for their monitoring. Considering its noninvasive character, as well as the accessibility and cost-effectiveness of the above technique, it may become a fairly promising tool for early diagnostics and monitoring of voice disturbances after thyroid surgery.

The present work is a pilot study, and its results must be confirmed by investigation on a large group of patients. Several questions remain to be answered and several technical problems await solution before we will be able to standardize the technique. However, we have decided to publish the obtained results as a pilot study outcome, assuming the potential of clinical applications of the presented technique. Further studies must concentrate on (1) definition of the VFDV cut off value for laryngeal nerve palsy diagnosis, (2) evaluation of the possibility of early discrimination between permanent and transitional RLN palsy, and (3) definition of the diagnostic criteria of EBSLN palsy by US technique.

\section{Conflicts of interest None.}

Open Access This article is distributed under the terms of the Creative Commons Attribution Noncommercial License which permits any noncommercial use, distribution, and reproduction in any medium, provided the original author(s) and source are credited.

\section{References}

1. Hirano M (1975) Phonosurgery: basic and clinical investigations. Otologia Fukuoka 21(Supp11):239-262

2. Hirano M (1974) Morphological structure of the vocal cord as a vibrator and its variations. Folia Phoniatr 26:89-94

3. Titze IR (1988) The physics of small-amplitude oscillation of the vocal folds. J Acoust Soc Am 83:1536-1552

4. Chevallier P, Marcy PY, Arens C, Raffalli C, Padovani B, Bruneton JN (2002) Larynx and Hypopharynx. In: Bruneton JN (ed) Applications of sonography in head and neck pathology. Springer, New York, pp 165-191

5. Hsiao TY, Wang CL, Chen CN, Hsieh FJ, Shau YW (2001) Noninvasive assessment of laryngeal phonation function using color Doppler ultrasound imaging. Ultrasound Med Biol 27:10351040

6. Shau YW, Wang CL, Hsieh FJ, Hsiao TY (2001) Noninvasive assessment of vocal fold mucosal wave velocity using color doppler imaging. Ultrasound Med Biol 27:1451-1460 
7. Raghavendra BN, Horii SC, Reede DL, Rumancik WM, Persky M, Bergeron T (1987) Sonographic anatomy of the larynx, with particular reference to the vocal cords. J Ultrasound Med 6:225-230

8. Miles KA (1989) Ultrasound demonstration of vocal cord movements. British J Radiol 62:871-872

9. Garel C, Legrand I, Elmaleh M, Contencin P, Hassan M (1990) Laryngeal ultrasonography in infants and children: anatomical correlation with fetal preparations. Pediatr Radiol 20:241-244

10. Ooi LL, Chan HS, Soo KC (1995) Color Doppler imaging for vocal cord palsy. Head Neck 17:20-23

11. Tsai CG, Chen JH, Shau YW, Hsiao TY (2009) Dynamic B-mode ultrasound imaging of vocal fold vibration during phonation. Ultrasound Med Biol 35:1812-1818

12. Isshiki N, Ohkawa M, Goto M (1985) Stiffness of the vocal cord in dysphonia-Its assessment and treament. Acta Otolaryngol (Stockh) 419:167-174 (Suppl)
13. Berke GS, Smith ME (1992) Intraoperative measurement of the elastic modulus of the vocal fold. Part 2. Preliminary results. Laryngoscope 102:770-778

14. Sercarz JA, Berke GS, Ming Y, Gerratt BR, Natividad M (1992) Videistroboscopy of human vocal fold paralysis. Ann Otol Rhinol Laryngol 101:567-577

15. Hanson DG, Jiang J, D'Agostino M, Herzon G (1995) Clinical measurement of mucosal wave velocity using simultaneous photoglottography and laryngostroboscopy. Ann Otol Rhinol Laryngol 104:340-349

16. Schindler O, Gonella ML, Pisani R (1990) Doppler ultrasound examination of the vibration speed of vocal folds. Folia Phoniatr 42:265-272

17. Friedman EM (1997) Role of ultrasound in the assessment of vocal cord function in infants and children. Ann Otol Rhinol Laryngol 106:199-209 